\title{
PREFACE: SELECTED IHMTC 2019 CONFERENCE PAPERS
}

This issue of the Journal of Enhanced Heat Transfer [JEH(T)] contains a collection of six extended papers from the 25th National and 3rd International ISHMT-ASTFE Heat and Mass Transfer Conference (IHMTC 2019).

IHMTC 2019 is a biennial international conference organized by the Indian Society of Heat and Mass Transfer (ISHMT). The ISHMT acts as a nodal agency in India, stimulating researchers engaged in various areas of heat and mass transfer and bringing them together for fruitful interaction and exchange of ideas. The ISHMT organized the first National Heat and Mass Transfer Conference at the Indian Institute of Technology Madras in 1971, and subsequently the conference has been held every two years at different places across the country. In these conferences, many well-known experts from abroad have participated, exchanging technical information and sharing their expertise with Indian researchers. In order to foster greater interaction with researchers from overseas, ISHMT has started collaborating with other premier professional societies. ISHMT and the American Society of Mechanical Engineers jointly organized this conference from 1994 to 2013. Thereafter, ISHMT and the American Society of Thermal and Fluids Engineers (ASTFE) have jointly organized this conference.

IHMTC 2019 was held in IIT Roorkee, India, and attracted a total of 950 regular paper submissions, spanning numerous active and emerging topic areas of heat and mass transfer. The conference program committee selected 647 regular papers to be presented at the conference and published in the conference proceedings.

The six extended papers for this special issue were selected from among all the accepted papers by special issue guest editor Arup Kumar Das, based on relevance to the journal and reviews of the conference version of the papers. The authors were asked to revise the conference paper for journal publication and, in accordance with customary practice, to add 30\% new material. The revised papers again went through the normal journal-style review process and finally are presented to the readers in the present form. We appreciate the willingness of the authors to help organize this special issue.

The six extended papers in this special issue cover entity detection and content extraction from web data, datatype inferring for RDF document matching, and $k$-core-truss discovering on graph data. In "Effect of Short Pin Fin with Different Shapes and Arrangements on Thermal Resistance of Micro Heat Sink," authors characterize thermohydraulics of short pin fins as heat sink through their experimental and numerical efforts. They concluded that the shape of the micro pin fin has the most important control on thermal resistance of the heat sink. Continuing along the same line, in "Enhanced Heat Transfer and Flow Features in a Duct Mounted with Various Ribs," authors experimented on ribbed passage to show the information about flow structures and explain the reason of variation in heat transfer distribution inside four types of rib configurations. In "Enhancement of Heat Transfer during Evaporation of R407C by Providing Intelligently Designed Structures Inside Tubes," authors proposed new designs of structures inside tubes for enhancement of heat transfer coefficient in ecofriendly refrigerant without pressure drop penalty. They compared the performance of the designed tubes with commercially available plain and microfin tubes. In "Amphiphilic Additives Enhanced Pool Boiling Heat Transfer in Confined Spaces," authors used amphiphilic additives such as surfactants and ionic liquids to 
enhance boiling heat transfer of water for downward-facing heaters. They reported nearly 2.2 times enhancement in heat transfer coefficient and almost 3.8 times increase in critical heat flux after adding these amphiphilic additives in water. In "An Experimental Investigation of Heat Transfer Performance of Wavy Channels under Laminar Flow Conditions: An Interferometric Study," authors evaluated the thermal performance factor of wavy channels of different phase shifts to propose enhancement of heat transfer for a range of Reynolds numbers. In "Wetting Front Studies on Heated Plate with Orthogonal Water Jet Impingement," authors showed water jet impingement cooling of a heated surface to establish that the wetting front growth increases with an increase in water flow rate and decreases with an increase in initial plate temperature.

From the six (extended) papers, we observe that the ISHMT community is actively engaged in research of enhancement in heat transfer. Society members are currently investigating a wide range of subject areas along the line of heat and mass transfer. We hope that readers enjoy this special issue.

I would like to thank all the conference authors and presenters. Additional thanks go out to the volunteers of the Technical Program Committee, whose efforts make these types of conferences possible. Lastly, I would like to thank the Society Chair, Prof. Pradip Dutta, IISC Bangalore, for all his encouragement and support. I would like to acknowledge efforts of the members of the local organizing committee, especially Prof. Ravi Kumar and Prof. Sudhakar Subudhi, for fantastic organization of the conference. The editor appreciates the timely efforts of the journal staff to follow up on the required actions and make this special issue happen.

Guest Editor

Arup Kumar Das

Department of Mechanical and Industrial Engineering

IIT Roorkee, India 